\title{
IT formulae for gamma target: mutual information and relative entropy
}

\author{
Benjamin Arras and Yvik Swan.
}

\begin{abstract}
In this paper, we introduce new Stein identities for gamma target distribution as well as a new non-linear channel specifically designed for gamma inputs. From these two ingredients, we derive an explicit and simple formula for the derivative of the input-output mutual information of this non-linear channel with respect to the channel quality parameter. This relation is reminiscent of the well-known link between the derivative of the input-output mutual information of additive Gaussian noise channel with respect to the signal-to-noise ratio and the minimum mean-square error. The proof relies on a rescaled version of De Bruijn identity for gamma target distribution together with a stochastic representation for the gamma-specific Fisher information. Finally, we are able to derive precise bounds and asymptotics for the input-output mutual information of the non-linear channel with gamma inputs.
\end{abstract}

Index Terms-Non-linear Channel, Mutual Information, Relative Entropy, Fisher Information, Estimation Theory.

\section{INTRODUCTION}

$\mathbf{L}$ Et $X, Y$ be two random variables on the same probability space, with joint probability measure $P^{X, Y}$ and marginals $P^{X}$ and $P^{Y}$, respectively. We choose the law of the couple $(X, Y)$ to be absolutely continuous with respect to a common dominating measure $\mu$ and denote $p^{X, Y}(x, y), p^{X}(x)$ and $p^{Y}(y)$ the corresponding Radon-Nikodym derivatives. The mutual information between $X$ and $Y$ is

$$
I(X ; Y)=E\left[\log \left(\frac{p^{X, Y}(X, Y)}{p^{X}(X) p^{Y}(Y)}\right)\right] .
$$

Mutual information satisfies $I(X ; Y) \geq 0$ with equality if and only if $X$ and $Y$ are independent and therefore mutual information captures the dependence between $X$ and $Y$. The relative entropy (a.k.a. Kullback-Leibler divergence) from $Y$ to $X$ is

$$
D(X \| Y)=E\left[\log \left(\frac{p^{X}(X)}{p^{Y}(X)}\right)\right] .
$$

Relative entropy satisfies $D(X \| Y) \geq 0$ with equality if and only if $X={ }^{\mathcal{L}} Y$ and therefore $\bar{D}(X \| Y)$ captures the difference between $\mathcal{L}(X)$ and $\mathcal{L}(Y)$. One speaks of Gaussian relative entropy (and then, often, of the relative entropy of $X$ only) if $Y$ is standard Gaussian.

Benjamin Arras is with the Laboratoire Jacques-Louis Lions, Universite Pierre et Marie Curie, 4 place Jussieu, 75005 Paris, e-mail: arrasbenjamin@gmail.com. His research was supported by a Welcome Grant from Université de Liège, Sart-Tilman, Allée de la découverte 12, B-8000 Liège, Belgium.

Yvik Swan is with the Department of mathematics of Université de Liège, Sart-Tilman, Allée de la découverte 12, B-8000 Liège, Belgium e-mail: yswan@ulg.ac.be. Yvik Swan gratefully acknowledges support by the Fonds de la Recherche Scientifique - FNRS under Grant MIS F.4539.16.

Copyright (c) 2017 IEEE
Mutual information and relative entropy are crucial in a wide variety of fields (see e.g. [40] for an overview) but are both generally analytically, and even in some cases algorithmically, intractable. It is thus useful to dispose of formulas allowing to control them in terms of quantities which are more amenable to computations. Two such formulas are Stam's De Bruijn identity [36], [8] and Guo, Shamai and Verdú's MMSE identity [13] (which we shall refer to as GSV identity in the sequel). Exact statements of these identities are deferred to Section III. Informally, the De Bruijn identity provides an explicit link between the Gaussian relative entropy of an absolutely continuous random variable $X$ and the Fisher information of $X$. Similarly, the GSV identity provides an explicit link between the mutual information in a Gaussian channel and the minimal mean square error in said channel. Both formulas are, as it turns out, essentially equivalent because either can be deduced - at least formally - from the other, see Sections II and VI They relate information theoretic quantities (relative entropy, mutual information) to quantities typically of interest in statistical estimation theory (Fisher information, MMSE) and have proven to be linchpins of important developments in contemporary information theoretic probability theory (e.g. for entropic CLTs [7], [18], [19], [4], [3], [39], analysis of additive Gaussian channels [24], [13] or, more generally, IT inequalities [15], [32]).

Both the De Bruijn and the GSV identities are obtained through a study of entropy/information jumps around $X$ along small perturbations of the form

$$
X \mapsto X_{r}:=\sqrt{r} X+N
$$

with $r>0$ and $N$ an independent standard Gaussian. The De Bruijn and GSV identities are thus, inherently, of a Gaussian nature and it is therefore natural to enquire whether similar relationships also hold outside of the Gaussian realm. Quoting [14], "a natural question to pose is how general the information-estimation relationship can be". This important question has of course already received a lot of attention in the literature and there exist De Bruijn identities, on the one hand, and GSV identities, on the other hand, for most classical target probability distributions of practical relevance (precise references will be given later in the text). The resulting identities, however, no longer enjoy the elegance and ease of manipulation of their Gaussian counterparts. In particular the estimation quantities derived in either cases do not bear natural interpretations and, to the best of our knowledge, the equivalence between the general-target De Bruijn and the general-target GSV identities has never been investigated. 
A first intuitive way to branch outside of the Gaussian scope is to work as in [14], [30] and extend [3] by considering more general (additive or even non-additive) noising mechanisms of the form

$$
X \mapsto X_{a}=h(a ; X ; W)
$$

where $h(\cdot ; \cdot ; \cdot)$ is a deterministic function, $a$ is a real parameter and $W$ is an independent noise following some arbitrary distribution. As could be expected, the classical MMSE quantity from estimation theory no longer plays any role in these identities, and the corresponding correct object is expressed as the correlation of two generally intractable conditional expectations (depending on log-derivatives of the density of $X_{a}$ ) which bears no explicit representation nor interpretation.

The random perturbation $X \mapsto X_{r}$ defined in (3) is known in the IT community as an "additive Gaussian channel", see [40]. Rescaled versions of this transformation, namely

$$
\sqrt{\tau} X+\sqrt{1-\tau} N, \quad \tau \in(0,1)
$$

have long been studied in the probabilistic literature, either under the name "smart path" [27] or, more classically, "Ornstein-Uhlenbeck evolute" around $X$ [6]. The key fact here is that the deformation $x \mapsto \sqrt{r} x+N$ arises naturally through the action of the heat semigroup and $X_{r}$ ought to be interpreted as a rescaled stochastic representation for the "smart path" interpolation between the law of $X$ and the Gaussian distribution. A general take on this semi-group interpretation leads to De Bruijn-type formulas with general reference probability measure (see [5], [6]) providing a direct link between the relative entropy $D(X \| Z)$ from a target random variable $Z$ to a random variable $X$ and a target-specific Fisher information structure. This Fisher information structure is, in general, implicit as it depends on the distribution of the ad hoc deformation $X_{r}$ which bears no explicit stochastic representation equivalent to (3).

In this paper we derive a new set of De Bruijn/GSV identities specifically when the target distribution is in the family of gamma distributions (which encompass as particular cases the chi-square and exponential distributions). There are two main new ingredients behind our results. The first ingredient is a family of Stein identities for gamma target distribution. Stein identities are characterizations of probability distributions through the action of target-specific differential operators (see e.g. the Gaussian Stein identity (10)). They are available for virtually any probability distribution allowing a closed form distribution ([21]) and are known to lie at the boundary between IT and estimation theory [9], [31], [33], [34], [22], [29], [28]. The second ingredient is a new noisingchannel $r \mapsto X_{r}$ specifically designed for gamma input (see (43)):

$$
X_{r, \alpha, \lambda}=\gamma(\alpha-1 / 2, \lambda)+\left(\sqrt{r X}+\frac{N}{\sqrt{2 \lambda}}\right)^{2},
$$

where $\alpha \geq 1 / 2, \lambda>0, r>0, X$ is the input distribution, $N$ is a standard normal random variable, $\gamma(\alpha-1 / 2, \lambda)$ a gamma random variable with parameters $(\alpha-1 / 2, \lambda)$ and $\{X, N, \gamma(\alpha-1 / 2, \lambda)\}$ are independent. Interestingly this channel is quadratic rather than linear as in (3). Also, in perfect analogy with the Gaussian case and contrarily to the noising mechanisms (4), this channel bears a natural probabilistic interpretation because there is a Markov process naturally associated with the gamma channel, namely the Laguerre process. This realisation is due to [2, Corollary 10], where the interpolation scheme along the Laguerre semigroup is shown to admit the following representation in law:

$$
\begin{aligned}
\forall \tau \in(0,1), X_{\tau}^{\alpha, \lambda}= & (1-\tau) \gamma(\alpha-1 / 2, \lambda)+(\sqrt{\tau} \sqrt{X} \\
& \left.+\sqrt{\frac{1-\tau}{2 \lambda}} N\right)^{2},
\end{aligned}
$$

where $X_{\tau}^{\alpha, \lambda}$ is a time-changed Laguerre process with initial law $X$ and $\{X, \gamma(\alpha-1 / 2, \lambda), N\}$ as previously. The appearance of the Laguerre process in this context should come as no surprise as it plays, with respect to the gamma law, an equivalent role to that of the Ornstein-Uhlenbeck process in the case of Gaussian perturbations. We mention that the Laguerre process is also important in the theory of real-valued symmetric diffusions (see [26], [6]), in mathematical finance under the name of CIR process (see [10], [11], [1]) and in the theory of continuous state branching processes with immigration (see [20], [23]).

It is not the first time that non-linear channel models have been studied in the information theory literature, particularly in connection with the gamma distribution. In [35], the authors introduced a non-linear noncentral chi-channel in order to model the channel law for certain solitons transmitted through nonlinear optical fibre. In particular, they obtained the following representation of the channel law (see their equation (9)):

$$
Y^{2}=\frac{1}{2} \sum_{i=1}^{4}\left(\frac{X}{\sqrt{2}}+N_{i}\right)^{2},
$$

with $\left\{N_{i}\right\}$ a collection of independent standard normal random variables. Note that this representation is very close to the Laguerre interpolation scheme when $\alpha$ is a half-integer (see Proposition 9 in [2]). In particular, it follows directly from [2] that our quadratic gamma channel admits the following representation in law when $\alpha=p / 2$ :

$$
X_{r, p, \lambda}=\sum_{i=1}^{p}\left(\sqrt{r} \sqrt{\frac{X}{p}}+\frac{N_{i}}{\sqrt{2 \lambda}}\right)^{2} .
$$

Moreover, they obtained a capacity lower bound (see Theorem 2 of [35]) very close to the bounds obtained in this paper (see our equations (60) and 69) for specific input distribution. Thus, this suggests that our quadratic gamma channel might be of interest in the modeling of information transmission in nonlinear transmission devices such as nonlinear optical fibre. In [17], the authors obtained general relations between information and estimation quantities in discrete-time Levy channels. In particular, they considered a gamma channel where the distribution of the output conditioned to the input is a gamma law with some deterministic shape parameter and scale parameter (see Definition 7 of [17]). Note that our definition of a quadratic gamma channel is different and that the law of the output conditioned to the input of our channel is linked to the non-central gamma distribution. 
The results from [2] confirm the claim that, in the case of a gamma target distribution, the triplet "optimal Gaussian perturbation / Ornstein Uhlenbeck process / additive Gaussian channel (3)" is to be replaced by "optimal gamma perturbation / Laguerre process / quadratic channel (6)". A question that this analogy raises is whether the above mentioned De Bruijn and GSV identities - along with their many consequences still hold in the gamma context. The purpose of this note is to answer this question positively. By combining our new family of Stein identities and the new quadratic gamma channel we derive, via elementary arguments, tractable and interpretable gamma-specific De Bruijn and GSV identities. While the De Bruijn identity is in essence a rescaling of known results from our previous paper [2], the gamma-GSV identity we obtain is entirely new. We prove that our quadratic channel has properties which are strikingly similar to the additive Gaussian channel in terms of mutual information and its asymptotics for large values of the channel quality parameter.

\section{A. Outline of the paper}

In Section [II we review the relevant known results for Gaussian target. In Section III we provide the necessary IT and Stein identities for gamma target and we also recall the ad hoc gamma-specific De Bruijn identity (Theorem 2). In Section IV we discuss the main properties of the gamma-counterpart to the smart path (3) and in Section V (mainly Proposition 5) we provide the key ingredient of the paper, namely a new representation of the (gamma-specific) Fisher information in terms of a quantity reminiscent of the minimal mean square error at the heart of the GSV equality. In Section VI we show that the quantities we have introduced are indeed the missing link between IT and estimation theory with gamma target: we derive an explicit GSV formula for gamma target as well as fine upper bounds for the variation of the mutual information with respect to the channel quality parameter. The bounds are universal to the extent that they depend on the distribution of the input only through its mean and the estimation theoretical quantity put forward in Proposition 5. The only assumption needed on the input $X$ is the existence of finite $\alpha+4$ moment. Finally, for gamma input with parameters $(\alpha, \lambda)$, we obtain an inequality on the mutual information reminiscent of the Gaussian case and for $\alpha=1 / 2$, we obtain the exact asymptotic for large values of the channel quality parameter of the inputoutput mutual information.

\section{IT AND STEIN IDENTITIES FOR GAUSSIAN TARGET}

Let $N$ be a standard Gaussian random variable with pdf $\gamma(x)=(2 \pi)^{-1 / 2} e^{-x^{2} / 2}$. Stein's well-known identity [38], [37] states that:

$$
E[N \phi(N)]=E\left[\phi^{\prime}(N)\right] \text { for all } \phi \in \mathcal{F}(N)
$$

with $\mathcal{F}(N)$ the collection of absolutely continuous test functions $\phi: \mathbb{R} \rightarrow \mathbb{R}$ such that $\phi^{\prime} \in L^{1}(N)$. Moreover if another random variable $X$ also satisfies 10 then $X={ }^{\mathcal{L}} N$. We refer the reader to [27, Lemma 3.1.2] for a streamlined proof. Extending identity 10 to arbitrary target entices us to associate to any random variable $X$ with mean $\mu$ and variance $\sigma^{2}$ a random variable $\rho_{X}(X)$ defined (almost everywhere) through the identity:

$$
E\left[\rho_{X}(X) \phi(X)\right]=-E\left[\phi^{\prime}(X)\right] \text { for all } \phi \in \mathcal{F}(X)
$$

with $\mathcal{F}(X)$ the collection of absolutely continuous test functions $\phi: \mathbb{R} \rightarrow \mathbb{R}$ such that $\phi^{\prime} \in L^{1}(X)$. The random variable $\rho_{X}(X)$ defined a.e. by (11) is called the score of $X$; it is easy to see that if $X$ has differentiable density $p_{X}$ which cancels at the border of its support then $\rho_{X}(X)=\left.\frac{\mathrm{d}}{\mathrm{d} x} \log p_{X}(x)\right|_{x=X}$ satisfies (11). In particular from (10) we know that $\rho_{X}(X)=$ $-\frac{X-\mu}{\sigma^{2}}$ if and only if $X={ }^{\mathcal{L}} \sigma N+\mu$ (here and throughout we reserve the notation $N$ for a standard normal random variable). Conditions on the distribution of $X$ under which the score is well-defined have been thoroughly adressed in the literature and it is a well-known fact that the score is essentially unique in the sense that if a random variable $Y$ satisfies (11) with the same score as $X$ then $Y={ }^{\mathcal{L}} X$; see [37], [18], [21]

Applying (11) to the test function $\phi(x)=1$ we deduce that if $X$ admits a score then necessarily $E\left[\rho_{X}(X)\right]=0$. The second moment of the score plays a role in the standardized relative Fisher information

$$
\begin{aligned}
J_{\text {st }}(X) & =\sigma^{2} E\left[\left(\rho_{X}(X)+(X-\mu) / \sigma^{2}\right)^{2}\right] \\
& =\sigma^{2} E\left[\rho_{X}(X)^{2}\right]-1
\end{aligned}
$$

and it is well known that $J_{\text {st }}(X)=0$ if and only if $X={ }^{\mathcal{L}}$ $\sigma N+\mu$ (see e.g. [18], [19], [29]); in other words the second moment of the score suffices to characterize the distribution. The quantity $I(X)=E\left[\rho_{X}(X)^{2}\right]$ is the Fisher information of $X$ and, from previous consideration,s we know that $I(\sigma N+$ $\mu)=\frac{1}{\sigma^{2}}$.

Set, for simplicity, $\mu=0$ and $\sigma^{2}=1$. Then, as anticipated in the introduction, relative entropy (2) and standardized Fisher information (12) are related through the De Bruijn identity

$$
\frac{\mathrm{d}}{\mathrm{d} r} D\left(X_{r} \| N\right)=\frac{1}{2(1+r)}\left(r+\frac{1}{r} J_{s t}\left(X_{r}\right)\right),
$$

still with $X_{r}$ as in (3) (see [8], [18] for a proof of [13) solely under moment assumptions on $X$ ). Applying a conditional version of (10), we note how for all sufficiently regular test functions $\phi$ we also have

$$
\begin{aligned}
E\left[\left(E\left[\sqrt{r} X \mid X_{r}\right]-X_{r}\right) \phi\left(X_{r}\right)\right] & =-E\left[N \phi\left(X_{r}\right)\right] \\
& =-E\left[\phi^{\prime}\left(X_{r}\right)\right]
\end{aligned}
$$

from which we deduce the representation

$$
\rho_{r}\left(X_{r}\right)=\sqrt{r} E\left[X \mid X_{r}\right]-X_{r}
$$

for $\rho_{r}\left(X_{r}\right)$ the score of $X_{r}$. This in turn leads to the representation of standardized Fisher information:

$$
\frac{1}{r} J_{\mathrm{st}}\left(X_{r}\right)=1-(1+r) E\left[\left(X-E\left[X \mid X_{r}\right]\right)^{2}\right]
$$

which provides a connection between Fisher information (and hence relative entropy) with estimation theory's Minimal Mean Square Error

$$
\operatorname{MMSE}(X, Y)=E\left[(X-E[X \mid Y])^{2}\right] .
$$


Plugging (16) into (13) we obtain

$$
\frac{\mathrm{d}}{\mathrm{d} r} D\left(X_{r} \| N\right)=\frac{1}{2}\left(1-\operatorname{MMSE}\left(X, X_{r}\right)\right),
$$

which is equivalent to formula (66) of Theorem 5 page 7 of [13].

As already touched upon in the introduction, the original GSV formula from [13] links mutual information (1) and MMSE (17) through:

$$
\frac{\mathrm{d}}{\mathrm{d} r} I\left(X ; X_{r}\right)=\frac{1}{2} \operatorname{MMSE}\left(X, X_{r}\right) .
$$

We conclude this section by showing how to obtain (19) from (18); our argument relies on ideas from Section II-D of [13]. We stress that our method of proof is robust towards a change of channel, in the sense that we will show in Section VI how it can be transposed from the Gaussian to the gamma setting. For $r>0$ we first set $\tau(r)=r /(r+1)$ and introduce the random variables

$$
\begin{aligned}
& \tilde{X}_{\tau(r)}(x)=\sqrt{\tau(r)} x+\sqrt{1-\tau(r)} N \\
& X_{r}(x)=\sqrt{r} x+N
\end{aligned}
$$

with $x \in(-\infty,+\infty)$ and $N$, as above, an independent standard Gaussian. We also set $X_{r}=X_{r}(X)$ and $\tilde{X}_{\tau(r)}=$ $\tilde{X}_{\tau(r)}(X)$. For any deterministic functional, we denote by $E_{X}\left[F\left(\tilde{X}_{\tau(r)}(X)\right)\right]$ (similarly with $\left.X_{r}(X)\right)$ the following type of integral:

$$
E_{X}\left[F\left(\tilde{X}_{\tau(r)}(X)\right)\right]=\int p_{X}(x) F\left(\tilde{X}_{\tau(r)}(x)\right) d x .
$$

By standard arguments we know that $I\left(X, \tilde{X}_{\tau(r)}\right)=I\left(X, X_{r}\right)$ and

$$
I\left(X, \tilde{X}_{\tau(r)}\right)=E_{X}\left[D\left(\tilde{X}_{\tau(r)}(X) \| N\right)\right]-D\left(\tilde{X}_{\tau(r)} \| N\right),
$$

where $E_{X}[\cdot]$ denotes an expectation taken with respect to $X$. Using regularity arguments provided in $[8]$ in combination with the chain rule we easily obtain:

$$
\begin{aligned}
\frac{d}{d r}\left(I\left(X, X_{r}\right)\right)=\frac{1}{r(r+1)}( & E_{X}\left[J_{s t}\left(\tilde{X}_{\tau(r)}(X)\right)\right] \\
& \left.-J_{s t}\left(\tilde{X}_{\tau(r)}\right)\right) .
\end{aligned}
$$

We can finally conclude.

Proposition 1. [Theorem 1 in [13]] Identity (19) holds if $X$ is centered with $E\left[X^{2}\right]=1$.

Proof. First note how for all $x$ the random variable $\tilde{X}_{\tau(r)}(x)$ remains Gaussian so that straightforward computations lead to:

$$
J_{s t}\left(\tilde{X}_{\tau(r)}(x)\right)=\frac{1}{2} \frac{r\left(r+x^{2}\right)}{1+r},
$$

for all real $x$. Next, by scaling arguments, we get

$$
J_{s t}\left(\tilde{X}_{\tau(r)}\right)=(1+r) J_{s t}\left(X_{r}\right)-\frac{r^{2}}{2} .
$$

Moreover, using (16) we obtain

$$
\begin{aligned}
J_{s t}\left(\tilde{X}_{\tau(r)}\right) & =\frac{1}{2}\left[(1+r) r\left(1-\operatorname{MMSE}\left(X, X_{r}\right)\right)-r^{2}\right], \\
& =\frac{1}{2}\left[-r(1+r) \operatorname{MMSE}\left(X, X_{r}\right)+r\right]
\end{aligned}
$$

Combining everything together, we have:

$$
\begin{aligned}
\frac{d}{d r}\left(I\left(X, X_{r}\right)\right) & =\frac{1}{2 r(r+1)}\left(E\left[\frac{r\left(r+X^{2}\right)}{1+r}\right]\right. \\
& \left.+r(1+r) \operatorname{MMSE}\left(X, X_{r}\right)-r\right) \\
& =\frac{1}{2} \operatorname{MMSE}\left(X, X_{r}\right),
\end{aligned}
$$

as required.

\section{IT AND STEIN IDENTITIES FOR GAMMA TARGET}

Let $Z$ be a gamma distributed random variable with pdf $\gamma_{\alpha, \lambda}(x)=\lambda^{\alpha} / \Gamma(\alpha) x^{\alpha-1} \exp (-\lambda x)$ over the positive half line. The equivalent of Stein's identity (10) for a gamma target has long been known to be

$$
E[(\lambda Z-\alpha) \phi(Z)]=E\left[Z \phi^{\prime}(Z)\right]
$$

(see [25]). Moreover if some positive random variable $X$ also satisfies this identity over an appropriately wide class of functions then $X={ }^{\mathcal{L}} Z$, see [12] for a proof. Introducing the derivative $\partial_{x}^{\sigma} \phi(x)=(\sqrt{x} \phi(x))^{\prime}$, we rewrite (28) as

$$
E[\sqrt{Z}(\lambda \sqrt{Z}-(\alpha-1 / 2) / \sqrt{Z}) \phi(Z)]=E\left[\sqrt{Z} \partial_{x}^{\sigma} \phi(Z)\right]
$$

for all $\phi \in \mathcal{F}(Z)$, with $\mathcal{F}(Z)$ a collection of sufficiently smooth test function $\phi: \mathbb{R}_{+}^{*} \rightarrow \mathbb{R}$ such that $x \mapsto \sqrt{x} \partial_{x}^{\sigma} \phi(x) \in$ $L^{1}(Z)$. While in appearance less elegant than (28), we claim that 29] is actually the correct starting point for Stein/IT analysis with a gamma target.

As in Section II we begin by extending the scope of 29. to arbitrary target by introducing for arbitrary positive $X$ a random variable $\rho_{X}^{\gamma}(X)$ defined (almost everywhere) through the identity:

$$
E\left[\sqrt{X} \rho_{X}^{\gamma}(X) \phi(X)\right]=-E\left[\sqrt{X} \partial_{x}^{\sigma} \phi(X)\right]
$$

for all $\phi \in \mathcal{F}^{\sigma}(X)$, with $\mathcal{F}^{\sigma}(X)$ the collection of absolutely continuous test functions $\phi: \mathbb{R} \rightarrow \mathbb{R}$ such that $x \mapsto \sqrt{x} \partial_{x}^{\sigma} \phi(x) \in L^{1}(X)$. We call $\rho_{X}^{\gamma}(X)$ defined by 30 . $X$ 's $\gamma$-score. Taking $\phi(x)=1 / \sqrt{x}$ in (30) we conclude that if $X$ admits a $\gamma$-score then necessarily it satisfies $E\left[\rho_{X}^{\gamma}(X)\right]=0$. From 29] we know that the $\gamma(\alpha, \lambda)$ distribution is characterized by

$$
\rho_{Z}^{\gamma}(Z)=-(\lambda \sqrt{Z}-(\alpha-1 / 2) / \sqrt{Z})
$$

Mimicking the Gaussian situation from Section $\Pi$ it is natural to measure distance to the gamma by comparing $\gamma$-scores with those in 31. 
Definition 1 (Standardized gamma Fisher information). The standardized $\gamma(\alpha, \lambda)$-Fisher information of a positive random variable $X$ with finite mean and $p d f p$ is:

$$
J_{\mathrm{st}, \gamma(\alpha, \lambda)}(X)=\frac{1}{\lambda} E\left[\left(\rho_{X}^{\gamma}(X)+\lambda \sqrt{X}-\frac{\alpha-1 / 2}{\sqrt{X}}\right)^{2}\right] .
$$

Standardized gamma Fisher information is not location invariant (we need the input to be positive) but behaves nicely under scaling (under the assumption that $E[X]=\alpha / \lambda$ ):

$$
\begin{aligned}
J_{\mathrm{st}, \gamma(\alpha, \lambda)}(a X)=J_{\mathrm{st}, \gamma(\alpha, a \lambda)}(X)= & \frac{1}{a} J_{\mathrm{st}, \gamma(\alpha, \lambda)}(X) \\
& +\alpha \frac{(a-1)^{2}}{a} .
\end{aligned}
$$

Note that (by straightforward integration by parts starting from (30)

$$
\sqrt{X} \rho_{X}^{\gamma}(X)=X \rho_{X}(X)+\frac{1}{2}
$$

with $\rho_{X}(x)=\left(\log p_{X}(x)\right)^{\prime}$ the usual score of $X$ (here we abuse notations slightly w.r.t. the definitions from Section [II]. Hence we can rewrite 32 as

$$
J_{\text {st }, \gamma(\alpha, \lambda)}(X)=\frac{1}{\lambda} E\left[X\left(\rho_{X}(X)+\lambda-(\alpha-1) / X\right)^{2}\right]
$$

which is precisely the relative Fisher information advocated by [6]. Aiming at a Cramer-Rao inequality one might wish to expand the square in 32 in order to identify the correct gamma-Fisher information, but it is easy to realize that this will not yield good results. Following [2] we rather propose to introduce

$$
I_{\gamma(\alpha, \lambda)}^{r}(X)=\frac{1}{\lambda} E\left[X\left(\rho_{X}(X)+\lambda(1+r)-\frac{(\alpha-1)}{X}\right)^{2}\right] .
$$

which we call a $r$-corrected gamma Fisher information. Clearly $I_{\gamma(\alpha, \lambda)}^{r}(Z)=\alpha r^{2}$ for all $r \geq 0$ and all $\lambda>0$ if $Z \sim \gamma_{\alpha, \lambda}$ (recall that $\rho_{Z}(Z)=(\alpha-1) / Z-\lambda$ in this case) and simple computations show that

$$
J_{\text {st }, \gamma(\alpha, \lambda)}(X)=I_{\gamma(\alpha, \lambda)}^{r}(X)-\alpha r^{2} \geq 0
$$

(we stress the important fact that this decomposition holds solely under a first moment assumption on $X$, see also [2, Remark 13]).

The relative entropy with respect to the gamma distribution is defined exactly as in the Gaussian case (recall (2)):

$$
D(X \| \gamma(\alpha, \lambda))=\int_{0}^{+\infty} p_{X}(u) \log \left(p_{X}(u) / \gamma_{\alpha, \lambda}(u)\right) d u
$$

with $X$ a random variable with density $p_{X}$ on the positive real line. Note how gamma relative entropy does not behave as Gaussian relative entropy under scaling:

$$
D(a X \| \gamma(\alpha, \lambda))=D(X \| \gamma(\alpha, a \lambda))
$$

for all $a>0$. There exists a De Bruijn identity specifically for [38), first identified by [5], [6] in the context of probability semigroup theory and $\Gamma$-calculus. We state a rescaling of the identity in its most general form as due to [2].
Theorem 2 (Gamma De Bruijn identity). Let $\alpha \geq 1 / 2$ and suppose that $X$ is a random variable with finite $\alpha+4$ moments. Then

$$
\frac{\mathrm{d}}{\mathrm{d} r} D\left(X_{r} \| \gamma(\alpha, \lambda /(1+r))\right)=\frac{1}{r} J_{s t, \gamma(\alpha, \lambda)}\left(X_{r}\right)-\alpha \frac{r}{1+r}
$$

where

$$
X_{r}=\gamma\left(\alpha-\frac{1}{2}, \lambda\right)+\left(\sqrt{r X}+\frac{N}{\sqrt{2 \lambda}}\right)^{2}
$$

with $\gamma\left(\alpha-\frac{1}{2}, \lambda\right)$ an independent gamma distributed random variable with parameters $\alpha-1 / 2, \lambda$ and $N$ as before an independent standard Gaussian random variable. The integrated version is

$$
D(X \| \gamma(\alpha, \lambda))=\int_{0}^{\infty}\left(\frac{1}{r} J_{s t, \gamma(\alpha, \lambda)}\left(X_{r}\right)-\alpha \frac{r}{1+r}\right) d r .
$$

\section{A QUADRATIC GAMMA CHANNEL}

Equations (40) and (41) lead us to introducing the nonlinear gamma channel (with all notations as in Theorem 2)

$$
X \mapsto X_{r}\left(:=X_{r, \alpha, \lambda}\right)=\gamma(\alpha-1 / 2, \lambda)+\left(\sqrt{r X}+\frac{N}{\sqrt{2 \lambda}}\right)^{2}
$$

for $r>0$. We also introduce the notation

$$
Y_{r}=\sqrt{r X}+\frac{N}{\sqrt{2 \lambda}}
$$

Conditionally on $X$, the random variable $X_{r}$ is the independent sum of a gamma and a non-central chi-squared random variable. This is the main difference between our channel (43) and classical "dual" channels wherein the distribution of the output, conditionally on the signal, remains within the same family of distributions as the noise (such as for instance in Gaussian channels studied in Section III or Poisson channels [16]).

Exploiting the moment generating function of $X_{r}$ we obtain the following description of the channel.

Proposition 3. - If $X$ has moment generating function $M_{X}(\cdot)$ on $(0, a)$ then the moment generating function of $X_{r}$ is

$$
M_{r}(t)=\left(1-\frac{t}{\lambda}\right)^{-\alpha} M_{X}\left(\frac{r t}{1-\frac{t}{\lambda}}\right)
$$

on $(0, \lambda /(\lambda r / a+1))$

- In particular if $E[X]=\alpha / \lambda$ then

$$
E\left[X_{r}\right]=\frac{\alpha}{\lambda}+r E[X]=\frac{\alpha}{\lambda}(1+r) .
$$

- Let $\lambda_{r} \leq \lambda$. The output $X_{r}$ is itself gamma distributed with parameters $\left(\alpha, \lambda_{r}\right)$ if and only if the input is gamma distributed with parameters $\left(\alpha, \frac{1}{r}\left(\frac{1}{\lambda_{r}}-\frac{1}{\lambda}\right)\right)$.

Remark 4. An equivalent way to express the second point in Proposition 3 if $X$ is gamma distributed with parameters $\alpha, \lambda_{1}$ then $X_{r}$ is gamma distributed with parameters $\left(\alpha, \lambda_{r}\right)$ where $\lambda_{r}=\left(\frac{r}{\lambda_{1}}+\frac{1}{\lambda}\right)^{-1}$ for all $r>0$. 


\section{RELATIVE ENTROPY AND ESTIMATION THEORY}

Proposition 5. Let $\alpha \geq 1 / 2$ and suppose that $X$ is positive with finite mean. Define $X_{r}, Y_{r}$ as in (43), (44) and introduce the ratio

$$
\mathcal{V}_{r}(X)=\frac{Y_{r}}{\sqrt{X_{r}}}
$$

Then

$\rho_{r}^{\gamma}\left(X_{r}\right)+\lambda \sqrt{X_{r}}-(\alpha-1 / 2) / \sqrt{X_{r}}=E\left[\lambda \sqrt{r X} \mathcal{V}_{r}(X) \mid X_{r}\right]$

and

$$
J_{s t, \gamma(\alpha, \lambda)}\left(X_{r}\right)=\lambda E\left[E\left[\sqrt{r X} \mathcal{V}_{r}(X) \mid X_{r}\right]^{2}\right] .
$$

Remark 6. Note how in particular if $\alpha=1 / 2$ then (46) reduces to $\operatorname{sign}\left(Y_{r}\right)$, the sign of $\sqrt{r X}+N / \sqrt{2 \lambda}$. This quantity plays a central role in Stein type representations for gamma specific Fisher information as obtained in [2. Proposition 23].

Remark 7. An immediate consequence of (48), Jensen's inequality for conditional expectations and the fact that $\mathcal{V}_{r}(X) \mid \leq 1$ is the inequality

$$
J_{\text {st }, \gamma(\alpha, \lambda)}\left(X_{r}\right) \leq \lambda r E[X]
$$

for all $\lambda, r \geq 0$ and all $\alpha \geq 1 / 2$.

Combining (48) with the gamma-specific De Bruijn identity (40) we immediately obtain that if $X$ is a positive random variable with finite $\alpha+4$ moment then

$$
\begin{aligned}
\frac{\mathrm{d}}{\mathrm{dr}} D\left(X_{r} \| \gamma(\alpha, \lambda /(1+r))\right)= & \frac{\lambda}{r} E\left[E\left[\sqrt{r X} \mathcal{V}_{r}(X) \mid X_{r}\right]^{2}\right] \\
& -\alpha \frac{r}{r+1}
\end{aligned}
$$

for all $r>0$.

Example 8. Suppose that the input signal $X$ is gamma distributed with parameters $(\alpha, \lambda)$ so that $X_{r}$ follows a gamma law with parameters $(\alpha, \lambda /(1+r))$ for each $r>0$ (recall Remark (4). Then, thanks to (79), we have

$$
E\left[\sqrt{r X} \mathcal{V}_{r}(X) \mid X_{r}\right]=\frac{r}{1+r} \sqrt{X_{r}}
$$

so that $J_{\mathrm{st}, \gamma(\alpha, \lambda)}\left(X_{r}\right)=\frac{\alpha r^{2}}{r+1}$ and $\frac{\mathrm{d}}{\mathrm{dr}} D\left(X_{r} \| \gamma(\alpha, \lambda /(1+\right.$ $r)))=0$, as expected.

\section{Mutual INFORMation AND ESTIMATION THEORY}

We start by restating identity 21] (which actually holds true for any channels) in the present gamma-target context. Let $r>0, \tau(r)=r /(r+1)$ and introduce the random variables

$$
\begin{aligned}
\tilde{X}_{\tau(r)}(x) & =(1-\tau(r)) \gamma\left(\alpha-\frac{1}{2}, \lambda\right) \\
& +\left(\sqrt{\tau(r) x}+\frac{\sqrt{1-\tau(r)}}{\sqrt{2 \lambda}} Z\right)^{2}, \\
X_{r}(x) & =\gamma(\alpha, 1 / 2)+\left(\sqrt{r x}+\frac{N}{\sqrt{2 \lambda}}\right)^{2}
\end{aligned}
$$

for $x \in[0,+\infty)$. We also write $X_{r}=X_{r}(X)$ and $\tilde{X}_{\tau(r)}=$ $\tilde{X}_{\tau(r)}(X)$. Then $I\left(X, X_{r}\right)=I\left(X, \tilde{X}_{\tau(r)}\right)$ and

$$
\begin{aligned}
I\left(X, \tilde{X}_{\tau(r)}\right)= & E_{X}\left[D\left(\tilde{X}_{\tau(r)}(X) \| \gamma(\alpha, \lambda)\right)\right] \\
& -D\left(\tilde{X}_{\tau(r)} \| \gamma(\alpha, \lambda)\right),
\end{aligned}
$$

where $E_{X}[\cdot]$ denotes an expectation taken with respect to $X$ as in 20). Similarly as in Section [I] we also deduce from the gamma-De Bruijn identity (see Theorem 14 of [2]) as well as the chain rule for differentiation:

Lemma 9. Let $\alpha \geq 1 / 2, \lambda>0$ and $r>0$. If $X$ is almost surely positive with finite $\alpha+4$ moments and mean $E[X]=$ $\alpha / \lambda$ then

$$
\begin{aligned}
\frac{d}{d r}\left(I\left(X, X_{r}\right)\right)= & \frac{1}{r(r+1)}\left(E_{X}\left[J_{s t, \gamma(\alpha, \lambda)}\left(\tilde{X}_{\tau(r)}(X)\right)\right]\right. \\
& \left.-J_{s t, \gamma(\alpha, \lambda)}\left(\tilde{X}_{\tau(r)}\right)\right)
\end{aligned}
$$

We are now in a position to obtain the gamma counterpart to the GSV identity (19). However, as already pointed out, the problem with the quadratic gamma channel is that it is more difficult to compute directly $J_{s t, \gamma(\alpha, \lambda)}\left(\tilde{X}_{\tau(r)}(x)\right)$ because $\tilde{X}_{\tau(r)}(x)$ is not a gamma random variable but rather a non-central gamma whose explicit density is complicated to manipulate.

Proposition 10. Let $\alpha \geq 1 / 2, \lambda>0, r>0$ and $X$ be a positive random variable with finite $\alpha+4$ moment and mean equal to $\alpha / \lambda$. Then

$$
\begin{aligned}
\frac{d}{d r}\left(I\left(X, X_{r}\right)\right)= & \lambda\left(E_{X}\left[X E\left[E\left[\mathcal{V}_{r}(X) \mid X_{r}(X)\right]^{2}\right]\right]\right. \\
& \left.-E\left[E\left[\sqrt{X} \mathcal{V}_{r}(X) \mid X_{r}\right]^{2}\right]\right) .
\end{aligned}
$$

Remark 11. It should be clear that the previous result holds true even if $E[X] \neq \alpha / \lambda$. The proof is similar by using the general relation,

$$
\begin{aligned}
J_{s t, \gamma(\alpha, \lambda)}\left(\tilde{X}_{\tau(r)}\right)= & (1+r) J_{s t, \gamma(\alpha, \lambda)}\left(X_{r}\right)-2 r^{2} \lambda E[X] \\
& +\frac{\lambda r^{2}}{(1+r)}\left(\frac{\alpha}{\lambda}+r E[X]\right),
\end{aligned}
$$

instead of 81.

\section{A. An upper bound}

An immediate consequence of 56 and the fact that $\mathcal{V}_{r}(X) \mid \leq 1$ is the upper bound

$$
\frac{d}{d r}\left(I\left(X, X_{r}\right)\right) \leq \lambda\left(E[X]-E\left[E\left[\sqrt{X} \mathcal{V}_{r}(X) \mid X_{r}\right]^{2}\right]\right) .
$$

Note that (58) is very close to the Gaussian GSV identity (19). In particular when $X \sim \gamma_{\alpha, \lambda}$, using (51) and (58), we have:

$$
\frac{d}{d r}\left(I\left(X, X_{r}\right)\right) \leq \frac{\alpha}{1+r}
$$

which leads to the fine bound:

$$
I\left(X, X_{r}\right) \leq \alpha \log (1+r) .
$$


The previous bound should be compared with the corresponding formula (11) of [13] which is satisfied by the mutual information of the additive Gaussian channel with Gaussian input. When $X \sim \gamma_{\alpha, \nu}$, we have the bound:

$$
I\left(X, X_{r}\right) \leq \alpha \log \left(1+\frac{\lambda r}{\nu}\right) .
$$

\section{B. A lower bound for $\alpha=\frac{1}{2}$}

We set $\alpha=\frac{1}{2}$. Assume that the input is gamma distributed with parameters $(1 / 2, \lambda)$. By definition, the mutual information between $X$ and $X_{r}$ is equal to:

$I\left(X, X_{r}\right)=\int p_{X_{r} \mid X=x}(u, x) p_{X}(x) \log \left(\frac{p_{X_{r} \mid X=x}(u, x)}{p_{X_{r}}(u)}\right) d u d x$

Let us compute explicitly the ratio between $p_{X_{r} \mid X=x}(u, x)$ and $p_{X_{r}}(u)$ in order to provide a simple lower bound for the logarithm term in the previous expression. We have:

$$
\begin{aligned}
\frac{p_{X_{r} \mid X=x}(u, x)}{p_{X_{r}}(u)} & =\Gamma(\alpha) \frac{\lambda e^{-\lambda u} e^{-\lambda r x}\left(\frac{u}{r x}\right)^{\frac{\alpha-1}{2}} I_{\alpha-1}(2 \lambda \sqrt{u x r})}{u^{\alpha-1} e^{-\frac{\lambda u}{r+1}}\left(\frac{\lambda}{r+1}\right)^{\alpha}}, \\
& =\Gamma(\alpha)(1+r)^{\alpha} \frac{1}{\lambda^{\alpha-1}} e^{-\frac{\lambda r u}{r+1}} e^{-\lambda r x} \\
& \times \frac{I_{\alpha-1}(2 \lambda \sqrt{u x r})}{(u r x)^{\frac{\alpha-1}{2}}} .
\end{aligned}
$$

Moreover, since $I_{-1 / 2}(z)=\sqrt{2 / \pi} \cosh (z) / \sqrt{z}$, we obtain:

$$
\begin{aligned}
\frac{p_{X_{r} \mid X=x}(u, x)}{p_{X_{r}}(u)} & =\sqrt{\pi} \sqrt{(1+r)} \frac{1}{\lambda^{-\frac{1}{2}}} e^{-\frac{\lambda r u}{r+1}} e^{-\lambda r x} \\
& \times \frac{\cosh (2 \lambda \sqrt{u x r})}{\sqrt{\pi \lambda}}, \\
& =\sqrt{(1+r)} e^{-\frac{\lambda r u}{r+1}} e^{-\lambda r x} \cosh (2 \lambda \sqrt{u x r}), \\
& \geq \frac{1}{2} \sqrt{(1+r)} e^{-\frac{\lambda r u}{r+1}} e^{-\lambda r x} e^{2 \lambda \sqrt{u x r}}
\end{aligned}
$$

Using the monotonicity of the logarithm, we obtain:

$$
\begin{aligned}
\log \left(\frac{p_{X_{r} \mid X=x}(u, x)}{p_{X_{r}}(u)}\right) \geq & \log \left(\frac{1}{2} \sqrt{(1+r)} e^{-\frac{\lambda r u}{r+1}}\right. \\
& \left.\times e^{-\lambda r x} e^{2 \lambda \sqrt{u x r}}\right) .
\end{aligned}
$$

This inequality implies the following on the mutual information between $X$ and $X_{r}$ :

$$
\begin{aligned}
I\left(X, X_{r}\right) & \geq \frac{1}{2} \log (1+r)-\log (2)-\frac{\lambda r}{r+1} E\left[X_{r}\right]-r \lambda E[X] \\
& +2 \lambda \sqrt{r} E\left[\sqrt{X_{r}} \sqrt{X}\right] \\
& \geq \frac{1}{2} \log (1+r)-\log (2)-r \alpha-r \alpha \\
& +2 \lambda \sqrt{r} E\left[\left|\sqrt{r} X+\frac{Z \sqrt{X}}{\sqrt{2 \lambda}}\right|\right]
\end{aligned}
$$

Thus

$$
\geq \frac{1}{2} \log (1+r)-\log (2)-2 r \alpha+2 \alpha r=\frac{1}{2} \log (1+r)-\log (2),
$$

where we have used the fact $|x| \geq x, X$ and $Z$ are independent and $E[Z]=0$. This lower bound combined with the bound 60 implies that:

$$
\lim _{r \rightarrow+\infty} \frac{I\left(X, X_{r}\right)}{\frac{1}{2} \log (1+r)}=1
$$

Remark 12. - Thus, for $\alpha=1 / 2$ and for a gamma$(1 / 2, \lambda)$ distributed input, the mutual information between $X$ and the output $X_{r}$ exhibits the same asymptotic for large values of the channel quality parameter $r$ as the mutual information between the additive Gaussian channel and a Gaussian input.

- It would be nice to know if such an asymptotic is still true for $\alpha>1 / 2$ and a gamma- $(\alpha, \lambda)$ distributed input. More generally we ask the question: for which input distribution do we have the same type of asymptotic as in (70) for the mutual information? Such questions are related to the concept of "MMSE dimension", see [41].

\section{A. Proof of Proposition 3}

Identity (45) follows by independence as well as the fact that, conditionally on $X$, the random variable $(\sqrt{2 \lambda r X}+N)^{2}$ is noncentral chi square distributed with non-centrality parameter $\sqrt{2 \lambda r X}$. Hence

$$
\begin{aligned}
E\left[e^{t X_{r}}\right] & =\left(1-\frac{t}{\lambda}\right)^{-(\alpha-1 / 2)} E\left[e^{t\left(\sqrt{r X}+\frac{N}{\sqrt{2 \lambda}}\right)^{2}}\right] \\
& =\left(1-\frac{t}{\lambda}\right)^{-(\alpha-1 / 2)} \frac{E\left[e^{\frac{r t}{1-t / \lambda} X}\right]}{(1-t / \lambda)^{1 / 2}}
\end{aligned}
$$

which is defined as long as $t \leq \lambda$ and $r t /(1-t / \lambda) \leq a$. To see the next claim it suffices to notice that if $M_{r}(t)=$ $\left(1-t / \lambda_{r}\right)^{-\alpha}$ then necessarily

$$
M_{X}(t)=\left(1-\frac{t}{r}\left(\frac{1}{\lambda_{r}}-\frac{1}{\lambda}\right)\right)^{-\alpha}
$$

for $t$ sufficiently small.

\section{B. Proof of Proposition 5}

Identity (48) follows immediately from (47) and (32). To see (47) note how for all smooth test functions

$$
\begin{aligned}
E & {\left[\left(\rho_{r}^{\gamma}\left(X_{r}\right)+\lambda \sqrt{X_{r}}-(\alpha-1 / 2) / \sqrt{X_{r}}\right) \sqrt{X_{r}} \phi\left(X_{r}\right)\right] } \\
= & E\left[\rho_{r}^{\gamma}\left(X_{r}\right) \sqrt{X_{r}} \phi\left(X_{r}\right)\right]+\lambda E\left[X_{r} \phi\left(X_{r}\right)\right] \\
& -E\left[(\alpha-1 / 2) \phi\left(X_{r}\right)\right] \\
= & -E\left[\sqrt{X_{r}}\left(\frac{1}{2 \sqrt{X_{r}}} \phi\left(X_{r}\right)+\sqrt{X_{r}} \phi^{\prime}\left(X_{r}\right)\right)\right] \\
& +\lambda E\left[X_{r} \phi\left(X_{r}\right)\right]-E\left[(\alpha-1 / 2) \phi\left(X_{r}\right)\right] \\
= & -\alpha E\left[\phi\left(X_{r}\right)\right]-E\left[X_{r} \phi^{\prime}\left(X_{r}\right)\right]+\lambda E\left[X_{r} \phi\left(X_{r}\right)\right]
\end{aligned}
$$

Expanding (41) we can rewrite the third summand as

$$
\begin{aligned}
\lambda E\left[X_{r} \phi\left(X_{r}\right)\right] & =E\left[\lambda \gamma\left(\alpha-\frac{1}{2}, \lambda\right) \phi\left(X_{r}\right)\right]+E\left[\lambda r X \phi\left(X_{r}\right)\right] \\
& +\sqrt{2 \lambda} E\left[\sqrt{r X} N \phi\left(X_{r}\right)\right]+\frac{1}{2} E\left[N^{2} \phi\left(X_{r}\right)\right] .
\end{aligned}
$$


Applying (28) to the function $\gamma \mapsto \phi\left(\gamma+Y_{r}^{2}\right)$ we get

$$
\begin{aligned}
E\left[\lambda \gamma\left(\alpha-\frac{1}{2}, \lambda\right) \phi\left(X_{r}\right)\right] & =\left(\alpha-\frac{1}{2}\right) E\left[\phi\left(X_{r}\right)\right] \\
& +E\left[\gamma\left(\alpha-\frac{1}{2}, \lambda\right) \phi^{\prime}\left(X_{r}\right)\right] .
\end{aligned}
$$

Applying (10) to the function $n \mapsto n \phi\left(\gamma\left(\alpha-\frac{1}{2}, \lambda\right)+(\sqrt{r X}+\right.$ $n / \sqrt{2 \lambda})^{2}$ ) we get

$$
\frac{1}{2} E\left[N^{2} \phi\left(X_{r}\right)\right]=\frac{1}{2} E\left[\phi\left(X_{r}\right)\right]+E\left[N \phi^{\prime}\left(X_{r}\right) \frac{Y_{r}}{\sqrt{2 \lambda}}\right] .
$$

Applying (10) to $n \mapsto \sqrt{r X} \phi\left(\gamma\left(\alpha-\frac{1}{2}, \lambda\right)+(\sqrt{r X}+n / \sqrt{2 \lambda})^{2}\right)$ we get

$$
\sqrt{2 \lambda} E\left[\sqrt{r X} N \phi\left(X_{r}\right)\right]=E\left[\sqrt{r X} \phi^{\prime}\left(X_{r}\right) 2 Y_{r}\right] .
$$

Resuming from (73) we compute

$$
\begin{aligned}
& E\left[\left(\rho_{r}^{\gamma}\left(X_{r}\right)+\lambda \sqrt{X_{r}}-(\alpha-1 / 2) / \sqrt{X_{r}}\right) \sqrt{X_{r}} \phi\left(X_{r}\right)\right] \\
& =E\left[\left\{-\alpha+\left(\alpha-\frac{1}{2}\right)+\frac{1}{2}+\lambda r X\right\} \phi\left(X_{r}\right)\right] \\
& +E\left[\left\{-X_{r}+\gamma\left(\alpha-\frac{1}{2}, \lambda\right)+\sqrt{r X} 2 Y_{r}+N \frac{Y_{r}}{\sqrt{2 \lambda}}\right\} \phi^{\prime}\left(X_{r}\right)\right] \\
& =E\left[\lambda r X \phi\left(X_{r}\right)\right]+E\left[\sqrt{r X} Y_{r} \phi^{\prime}\left(X_{r}\right)\right] \\
& =\lambda E\left[\sqrt{r X}\left(\sqrt{r X}+\frac{N}{\sqrt{2 \lambda}}\right) \phi\left(X_{r}\right)\right],
\end{aligned}
$$

the last identity being a consequence of (77). By a standard density argument (identity (78) is valid for all smooth functions with compact support) we can then deduce the representation

$$
\begin{aligned}
\rho_{r}^{\gamma}\left(X_{r}\right)+ & \lambda \sqrt{X_{r}}-(\alpha-1 / 2) / \sqrt{X_{r}} \\
& =\frac{\lambda E\left[\sqrt{r X}\left(\sqrt{r X}+\frac{N}{\sqrt{2 \lambda}}\right) \mid X_{r}\right]}{\sqrt{X_{r}}} \\
& =\lambda E\left[\sqrt{r X} \mathcal{V}_{r}(X) \mid X_{r}\right]
\end{aligned}
$$

which leads to 47].

\section{Proof of Proposition 10}

First of all, applying Lemma 9 , we have:

$$
\begin{aligned}
\frac{d}{d r}\left(I\left(X, X_{r}\right)\right)= & \frac{1}{r(r+1)}\left(\int p_{X}(x) J_{s t, \gamma(\alpha, \lambda)}\left(\tilde{X}_{\tau(r)}(x)\right) d x\right. \\
& \left.-J_{s t, \gamma(\alpha, \lambda)}\left(\tilde{X}_{\tau(r)}\right)\right)
\end{aligned}
$$

Applying a slight extension of 33 to $\tilde{X}_{\tau(r)}=\frac{1}{r+1} X_{r}$ we deduce

$$
J_{s t, \gamma(\alpha, \lambda)}\left(\tilde{X}_{\tau(r)}\right)=(1+r) J_{s t, \gamma(\alpha, \lambda)}\left(X_{r}\right)-\alpha r^{2} .
$$

Applying Proposition 5 then leads to

$J_{s t, \gamma(\alpha, \lambda)}\left(\tilde{X}_{\tau(r)}\right)=(1+r) \lambda E\left[E\left[\sqrt{r X} \mathcal{V}_{r}(X) \mid X_{r}\right]^{2}\right]-\alpha r^{2}$.
Now, Proposition 5 is true irrespectively of the distribution of the input, so that we also have (for each fixed $x$ )

$$
J_{s t, \gamma(\alpha \lambda)}\left(X_{r}(x)\right)=\lambda E\left[E\left[\sqrt{r x} \mathcal{V}_{r}(x) \mid X_{r}(x)\right]^{2}\right] .
$$

Furthermore, we have

$$
\begin{aligned}
J_{s t, \gamma(\alpha, \lambda)}\left(\tilde{X}_{\tau(r)}(x)\right)= & (1+r) J_{s t, \gamma(\alpha, \lambda)}\left(X_{r}(x)\right) \\
& -2 r^{2} \lambda x+\frac{\lambda r^{2}}{(1+r)}\left(\frac{\alpha}{\lambda}+r x\right),
\end{aligned}
$$

which leads to

$$
\begin{aligned}
J_{s t, \gamma(\alpha, \lambda)}\left(\tilde{X}_{\tau(r)}(x)\right) & =(1+r) \lambda E\left[E\left[\sqrt{r x} \mathcal{V}_{r}(x) \mid X_{r}(x)\right]^{2}\right] \\
& -2 r^{2} \lambda x+\frac{\lambda r^{2}}{(1+r)}\left(\frac{\alpha}{\lambda}+r x\right) .
\end{aligned}
$$

Integrating the previous expression with respect to the density of $X$ together with the fact that $E[X]=\frac{\alpha}{\lambda}$, we obtain

$$
\begin{aligned}
(I) & :=\int_{\mathbb{R}_{+}^{*}} J_{s t, \gamma(\alpha, \lambda)}\left(\tilde{X}_{\tau(r)}(x)\right) p_{X}(x) d x \\
& =(1+r) \int_{\mathbb{R}_{+}^{*}} \lambda E\left[E\left[\sqrt{r x} \mathcal{V}_{r}(x) \mid X_{r}(x)\right]^{2}\right] p_{X}(x) d x \\
& -2 r^{2} \lambda \int_{\mathbb{R}_{+}^{*}} x p_{X}(x) d x+\frac{\lambda r^{2}}{1+r}\left(\frac{\alpha}{\lambda}+r \int_{\mathbb{R}_{+}^{*}} x p_{X}(x) d x\right), \\
& =(1+r) \int_{\mathbb{R}_{+}^{*}} \lambda E\left[E\left[\sqrt{r x} \mathcal{V}_{r}(x) \mid X_{r}(x)\right]^{2}\right] p_{X}(x) d x \\
& -2 r^{2} \alpha+\alpha r^{2}, \\
& =(1+r) \int_{\mathbb{R}_{+}^{*}} \lambda E\left[E\left[\sqrt{r x} \mathcal{V}_{r}(x) \mid X_{r}(x)\right]^{2}\right] p_{X}(x) d x \\
& -\alpha r^{2} .
\end{aligned}
$$

Combining 88 and 82 together with 80 , we obtain the relation:

$$
\begin{aligned}
\frac{d}{d r}\left(I\left(X, X_{r}\right)\right)= & \lambda\left(\int_{\mathbb{R}_{+}^{*}} x E\left[E\left[\mathcal{V}_{r}(x) \mid X_{r}(x)\right]^{2}\right] p_{X}(x) d x\right. \\
& \left.-E\left[E\left[\sqrt{X} \mathcal{V}_{r}(X) \mid X_{r}\right]^{2}\right]\right)
\end{aligned}
$$

leading directly to the claim.

\section{ACKNOWLEDGMENT}

We thank two anonymous referees and the Associate Editor for their pertinent remarks which have led to substantial improvement of the paper.

\section{REFERENCES}

[1] A. Alfonsi. Affine Diffusions and Related Processes: Simulation, Theory and Applications Springer, Vol. 6, 2015.

[2] B. Arras and Y. Swan. A stroll along the gamma. to appear in Stochastic Process. Appl., 2017.

[3] S. Artstein, K. Ball, F. Barthe and A. Naor. On the rate of convergence in the entropic central limit theorem. Probab. Theory Related Fields, 129(3):381-390, 2004.

[4] K. Ball, F. Barthe and A. Naor. Entropy jumps in the presence of a spectral gap. Duke Math. J., 119(1):41-63, 2003.

[5] D. Bakry and M. Emery. Diffusions hypercontractives. Séminaire de Probabilités, XIX:179-206, 1985. 
[6] D. Bakry, I. Gentil and M. Ledoux. Analysis and Geometry of Markov diffusion operators. Springer, 2014.

[7] A.R. Barron. Entropy and the Central Limit Theorem. Ann. Probab., 14(1):336-342, 1986

[8] A.R. Barron. Monotonic central limit theorem for densities. Technical Report, 50, Dept. Statistics, Stanford Univ.

[9] L. Brown, A. DasGupta, L. R. Haff and W.E. Strawderman. The heat equation and Stein's identity: Connections, applications. J. Statist. Plann. Inference, 136(7):2254-2278, 2006.

[10] J. C. Cox, J. E. Jr Ingersoll and S. A. Ross. An intertemporal general equilibrium model of asset prices. Econometrica, 53(2):363-384, 1985.

[11] J. C. Cox, J. E. Jr Ingersoll and S. A. Ross. A theory of the term structure of interest rates. Econometrica, 53(2):385-407, 1985.

[12] R.E. Gaunt, A.Pickett, and G. Reinert. Chi-square approximation by Stein's method with application to Pearson's statistic. Ann. Appl. Probab. 27(2):720-756, 2017

[13] D. Guo, S. Shamai, and S. Verdú. Mutual information and minimum mean-square error in Gaussian channels. IEEE Trans. Inform. Theory, 51(4):1261-1282, 2005.

[14] D. Guo, S. Shamai, and S. Verdú. Additive non-Gaussian noise channels: Mutual information and conditional mean estimation. Proceedings International Symposium on Information Theory (ISIT), 2005

[15] D. Guo, S. Shamai, and S. Verdú. Proof of entropy power inequalities via MMSE. Proceedings International Symposium on Information Theory (ISIT), 1011-1015, 2006

[16] D. Guo, S. Shamai, and S. Verdú. Mutual information and conditional mean estimation in Poisson channels. IEEE Trans. Inform. Theory, 54(5):1837-49, 2008

[17] J. Jiao, K. Ventkat, T. Weissman. Relations between Information and Estimation in Discrete-Time Levy Channels. to appear in IEEE Trans. Inform. Theory, 2017.

[18] O. Johnson. Information theory and the central limit theorem. Imperial College Press, London, 2004.

[19] O. Johnson and A.R. Barron. Fisher information inequalities and the central limit theorem. Probab. Theory Related Fields, 129:391-409, 2004.

[20] K. Kawazu and S. Watanabe. Branching processes with immigration and related limit theorems. Theory Probab. Appl., 16(1):36-54, 1971.

[21] C. Ley, G. Reinert and Y. Swan. Stein's method for comparison of univariate distributions. Probab. Surv., 14:1-52, 2017.

[22] C. Ley and Y. Swan. Stein's density approach and information inequalities. Electron. Comm. Probab, 18(7):1-14, 2013.

[23] Z. H. Li. Branching processes with immigration and related topics. Frontiers of Mathematics in China, 1(1):73-97, 2006.

[24] A. Lozano, A. M. Tulino and S. Verdú. Optimum power allocation for parallel Gaussian channels with arbitrary input distributions. IEEE Trans. Inform. Theory, 52(7):3033-3051, 2006.

[25] H. Luk. Stein's method for the Gamma distribution and related statistical applications. PhD thesis, University of Southern California, 1994.

[26] O. Mazet. Classification des Semi-Groupes de diffusion sur $\mathbb{R}$ Séminaire de Probabilités, XXXI:40-53, 1997.

[27] I. Nourdin and G. Peccati. Normal approximations with Malliavin calculus, volume 192 of Cambridge Tracts in Mathematics. Cambridge University Press, Cambridge, 2012. From Stein's method to universality.

[28] I. Nourdin, G. Peccati and Y. Swan. Entropy and the fourth moment phenomenon. J. Funct. Anal., 266:3170-3207, 2014.

[29] I. Nourdin, G. Peccati and Y. Swan. Integration by parts and representation of information functionals. IEEE International Symposium on Information Theory (ISIT), 2217-2221, 2014.

[30] D. P. Palomar and S. Verdu. Representation of mutual information via input estimates. IEEE Trans. Inform. Theory, 53(2):453-470, 2007.

[31] S. Park, S. Erchin and K. Qaraqe. On the equivalence between Stein and de Bruijn identities. IEEE Trans. Inform. Theory, 58(12):7045-7067, 2012.

[32] O. Rioul. Information theoretic proofs of entropy power inequalities. IEEE Trans. Inform. Theory, 57(1):33-55, 2011.

[33] I. Sason Improved lower bounds on the total variation distance for the Poisson approximation. Statist. Probab. Lett., 83(10):2422-2431, 2013.

[34] I. Sason, Entropy bounds for discrete random variables via maximal coupling. IEEE Trans. Inform. Theory, 59(11):7118-7131, 2013.

[35] N. A. Shevchenko, J. E. Prilepsky, S. A. Derevyanko, A. Alvarado, P. Bayvel and S. K. Turitsyn. A Lower Bound on the per Soliton Capacity of the Nonlinear Optical Fibre Channel. IEEE Information Theory Workshop - Fall (ITW), 104-108, 2015

[36] A. J. Stam. Some inequalities satisfied by the quantities of information of Fisher and Shannon. Inform. and Control, 2:101-112, 1959.
[37] C. Stein. Approximate computation of expectations. Institute of Mathematical Statistics Lecture Notes-Monograph Series, 7. Institute of Mathematical Statistics, Hayward, CA, 1986.

[38] C. M. Stein. Estimation of the mean of a multivariate normal distribution. Ann. Statist., 9(6):1135-1151, 1981.

[39] A.M. Tulino and S. Verdú. Monotonic decrease of the non-Gaussianness of the sum of independent random variables: A simple proof. IEEE Trans. Inform. Theory, 52(9):4295-4297, 2006.

[40] T. M. Cover and J. A. Thomas. Elements of Information Theory. John Wiley \& Sons, 2012

[41] Y. Wu and S. Verdú. MMSE dimension. IEEE Trans. Inform. Theory, 57(8):4857-4879, 2011.

Benjamin Arras received his M.Sc. degree in mathematics from Université Paris-Sud XI, France and his Ph.D. degree in applied mathematics from Ecole Centrale Paris, France in 2011 and 2014 respectively. After few months at Ceremade at Paris-Dauphine University, he joined the department of mathematics at Université de Liège in 2015 for one year. Then, he moved to the Laboratoire Jacques-Louis Lions at Université Pierre et Marie Curie, France in 2017. His main areas of research are stochastic calculus, fractional processes, Stein's method and weak limit theorems.

Yvik Swan was born in Dublin, Ireland, in 1979. He received a Master's degree in mathematics from the Université libre de Bruxelles, Brussels, in 2002, a DEA degree from the ISRO, Brussels, in 2004 and a Ph.D. degree in mathematical statistics from the Université libre de Bruxelles, Brussels, in 2007. From 2002 to 2008, he was Assistant at the mathematics department of the Université libre de Bruxelles, from 2008 to 2010 he was Chargé de Recherche at the Belgian National Fund for Scientific Research (FNRS), in 2011 and 2012 he was research assistant at the Université du Luxembourg and he is Junior tenured professor at the Université de Liège since 2013. He is the author of 25 articles. His research interests are currently mainly focused on the development of Stein's method for non Gaussian distributions. Dr. Swan is member of the BSS and BMS (Belgian and French Statistical Societies), is recipient of the "Prix du concours annuel de la classe des sciences de l'Académie Royale de Belgique" (prize received in 2011 for a thesis on Robbins' problem of optimal stopping) and is twice recipient of the Wernaers Prize for research and dissemination of science. 